\title{
A NOTE ON WEAKLY SEPARABLE SPACES
}

\section{R.B. Beshimov}

\begin{abstract}
We study the notion of a weak separable space. Some counterexamples, which show a strictness of previous results of the author, are given. The weak separability of hyperspaces and function spaces is investigated.
\end{abstract}

\section{Introduction}

Weakly separable spaces were introduced in [2]. In this article and in [3], [4], and [5] there were investigated some properties of weakly separable spaces, their relations with separable spaces, their behavior with respect to some topological constructions like topological products, continuous images, compactifications, covariant functors and so on. It should be mentioned also that in 1977 the weak separability property implicitely was considered by E.K. van Douwen in [8], where he studied density of compactifications of Tychonoff spaces.

Here we continue the investigation of the class of weakly separable spaces. First of all, we discuss Theorem 2.3, which states that every weakly separable, Hausdorff compact space is separable. It is shown that one cannot weaken neither separation axiom, nor compactness condition (Examples 3.1, 3.2 , and 3.4). We prove also that the weak separability property is preserved by certain functorial constructions in the class of $T_{1}$-spaces (Theorem 4.5 ). But this is not true for $T_{0}$-spaces (Example 4.6). Theorem 4.12 is a generalization of Shanin's theorem on a calibre. At last, we characterize spaces $X$ with weakly separable $C_{p}(X)$ (Theorem 5.3 ) and show that for some compacta $X$ spaces $C_{p}(X)$ are weakly separable, but non-separable (Example 5.4).

\section{Preliminaries}

Let us recall that a family $\mathcal{B}$ of non-empty (open) subsets of a topological space $X$ is said to be a $(\pi$-base $) \pi$-network of $X$ if for every non-empty open

AMS (MOS) Subject Classification 1991. Primary: 54A25, 54B20, 54C20, 54C35.

Key words and phrases: Weakly separable space, compact space, hyperspace, function space. 
set $U \subset X$ there is a set $V \in \mathcal{B}$ such that $V \subset U$. A family $\mathcal{B}$ of subsets of $X$ is called:

1) centered if $\cap \mathcal{B}_{0} \neq \emptyset$ for any finite $\mathcal{B}_{0} \subset \mathcal{B}$;

2) $\sigma$-centered if $\mathcal{B}=\cup\left\{\mathcal{B}_{i}: i \in \omega\right\}$, where each $\mathcal{B}_{i}$ is centered.

The next definition is due to V.I.Ponomarev.

2.1. Definition. A topological space $X$ is called weakly separable if $X$ has a $\sigma$-centered $\pi$-base.

2.2. Proposition. For an arbitrary topological space $X$ the following conditions are equivalent:

1) every $\pi$-base of $X$ is $\sigma$-centered;

2) $X$ is weakly separable;

3) $X$ has a $\sigma$-centered $\pi$-network;

4) every family $\mathcal{O}$ of non-empty open subsets of $X$ is $\sigma$-centered.

Proof. We need only to check the implication 3) $\rightarrow 4$ ). Take a $\sigma$-centered $\pi$-network $\mathcal{B}=\cup_{i=0}^{\infty} \mathcal{B}_{i}$. Set

$$
\mathcal{O}_{i}=\left\{U \in \mathcal{O}: \text { there is a } V \in \mathcal{B}_{i} \text { which is contained in } U\right\} .
$$

By definition of a $\pi$-network, $\mathcal{O}=\cup_{i=0}^{\infty} \mathcal{O}_{i}$. Since $\mathcal{B}_{i}$ is centered, $\mathcal{O}_{i}$ is centered too. Proposition 2.2 is proved.

Clearly, every separable space is weakly separable. Sometimes the reverse is true (look at [2] and [3]). One of main properties of the weak separability is contained in the next statement.

2.3. Theorem. A Hausdorff compact space $X$ is separable if and only if $X$ is weakly separable.

Let $X$ be a subspace of $Y$. We say that $Y$ is a topological extension of $X$, if $X$ is dense in $Y$.

2.4. Proposition. The class of weakly separable spaces is closed with respect to the following topological operations:

1) open subsets;

2) dense subsets;

3) topological extensions;

4) finite products;

5) countable unions;

6) continuous images.

The proof is rather simple and can be found in [2].

2.5. Theorem [5]. Every weakly separable space $X$ has a separable extension $e X$. 
2.6. Remark. The results of this section can be extended to an arbitrary cardinal number $\tau$. Namely, we say that the weak density of a space $X$ is no more than $\tau$ (write $w d(X) \leq \tau$ ) if $X$ has a $\pi$-base $\mathcal{B}$ which is a union of $\tau$ centered systems $\mathcal{B}_{\alpha}$. For example, Proposition 2.4 can be generalized by changing the class of all weakly separable spaces to the class of all spaces $X$ with $w d(X) \leq \tau$. A more general version of Theorem 2.3 can be written as:

$$
d(X)=w d(X)
$$

for an arbitrary Hausdorff compact space $X$.

\section{Some counterexamples}

3.1. Example. There exists a compact weakly separable $T_{1}$-space $X$ that is not separable.

Proof. As the underlying set for the space $X$ we take the space $Y=\omega_{1}$ of all countable ordinals. The topology of $X$ is a weakening of the topology of $Y$. Namely: non-empty open sets of $X$ are complements of compact subsets of $Y$. Clearly, these sets give us a topology on the set $X$. Since singletons are closed in this topology, $X$ is a $T_{1}$-space. It is evident, that topologies of spaces $X$ and $Y$ coincide on compact subsets of $Y$. Consequently, every centered system $\Phi$ of closed subsets of $X$ is a centered system of compact subsets of $Y$. Hence, $\Phi$ has a non-empty intersection. This implies a compactness of the space $X$.

Since each finite union of compact spaces is compact, the family of all non-empty open subsets of the space $X$ is centered. Thus, $X$ is weakly separable, because it has a centered base. It remains to show that $X$ is not separable. Let $X_{0}$ be an arbitrary countable subset of $X$. It is well known that in the space $Y=\omega_{1}$ the closure of every countable set is a (countable) compactum. Therefore, $X_{0}$ is contained in some compact subspace $F$ of the space $Y$. Let $U=Y \backslash F$. Then $U$ is a non-empty open subset of $X$, which doesn't meet $X_{0}$. Hence, $X_{0}$ is not dense in $X$. So, $X$ is not separable.

Example 3.1 shows us that we cannot weaken the Hausdorff separation axiom in Theorem 2.3. As for a compactness, one can weaken it to a local compactness in view of Proposition 2.4.1. But the following two examples show that the assertion of Theorem 2.3 cannot be extended neither to countably compact spaces, nor to Lindelof spaces. To describe these examples we would like to recall two definitions.

Let $\left\{X_{\alpha}: \alpha \in A\right\}$ be a collection of topological spaces, and let $a=\left(a_{\alpha}\right)$ be a point of their topological product $X$. By $\Sigma(a)$ we denote the subspace of $X$ consisting of all points $\left(x_{\alpha}\right)$ such that $x_{\alpha} \neq a_{\alpha}$ only for countably many 
$\alpha \in A$. The space $\Sigma(a)$ is called the $\Sigma$-product of the spaces $X_{\alpha}$ over the point $a$. A very important subspace of $\Sigma(a)$ is the set $\sigma(a)$ ( $\sigma$-product of $X_{\alpha}$ over $a$ ) consisting of all points $\left(x_{\alpha}\right)$ such that $x_{\alpha} \neq a_{\alpha}$ only for finitely many $\alpha \in A$.

If each $X_{\alpha}$ is a topological group, then both $\Sigma(e)$ and $\sigma(e)$, where $e=$ $\left(e_{\alpha}\right)$ and $e_{\alpha}$ is the neutral element of $X_{\alpha}$, are topological groups as well. The following example, as a topological group, is due to L.S. Pontryagin [12].

3.2. Example. There exists a normal, countably compact, weakly separable, but non-separable topological group $G$.

Let $D_{\alpha}=\left\{0_{\alpha}, 1_{\alpha}\right\}$ be a copy of the group $D=\{0,1\}=\mathbf{Z}_{2}$. For the group $G$ one can take the $\Sigma$-product of the groups $D_{\alpha}, \alpha \in \omega_{1}$, over the zero-point $0=\left(0_{\alpha}\right)$. In fact, normality of $G$ has been proved in [7]. Countable compactness of $G$ has been noticed by L.S. Pontryagin [12], who implicitely has introduced $\Sigma$-products of topological groups. At last, it has been noticed in [6], that $G$ is non-separable, but has a separable compactification.

3.3. Remark. What actually has been proved in [6] is, that the $\Sigma$-product of the continuum many groups $D_{\alpha}$ is not separable. But if we repeat this proof word for word, we shall prove non-separability of the group $G$ from Example 3.2.

3.4. Example. There exists a normal, Lindelof, weakly separable, but nonseparable topological group $H$.

For the group $H$, one can take the $\sigma$-product of the groups $D_{\alpha}, \alpha \in \omega_{1}$, over the zero-point. Since $H$ is dense subset of $G$, it is weakly separable by Proposition 2.4.2. Further, $H$ is not separable as a dense subset of the nonseparable group $G$. Finally, $H$ is a Lindelof space being a union of countably many compact sets $H_{n}$, where $H_{n}$ consists of all points $h=\left(h_{\alpha}\right)$ such that $h_{\alpha} \neq 0_{\alpha}$ at most for $n$ coordinates.

Let us recall that a Tychonoff space $X$ is said to be a $p$-space if there is a countable set of families $u_{i}, i \in \omega$, such that:

1) $u_{i}$ consists of open subsets of $\beta X$;

2) $X \subset \cup u_{i}$ for any $i \in \omega$;

3) $\cap\left\{S t\left(x, u_{i}\right): i \in \omega\right\} \subset X$ for any $x \in X$.

If in this definition each family $u_{i}$ consists of one open set, then we obtain definition of a Čech complete space.

3.5. Remark. The group $H$ from Example 3.4 is not a $p$-space. In fact, assume that $H$ is a $p$-space. Then there exists a perfect mapping $f: H \rightarrow Y$ onto a metric (separable) space $Y$ [1]. Take an arbitrary point $y \in Y$. Then 
$f^{-1}(y)$ is a $G_{\delta}$-compactum in $H$. But $H$ is dense in $D^{\omega_{1}}$. Hence, $f^{-1}(y)$ is a $G_{\delta^{-}}$-set in $D^{\omega_{1}}$. But no compact $G_{\delta^{-}}$-subset of $D^{\omega_{1}}$ is contained in $H$. We arrive at a contradiction.

In view of Example 3.4 and Remark 3.5 the next question is of some interest.

3.6. Question. Is it true that every weakly separable Lindelöf $p$-space is separable?

We cannot even answer the next question.

3.7. Question. Is it true that every weakly separable, Lindelöf, Čech complete space is separable?

3.8. Remark. It should be mentioned that both spaces from Examples 3.2 and 3.4 appeared also in [8] (Examples 3.3 and 3.1 respectively). The same is true about Questions 3.6 and 3.7 (see [8], Question 1).

Let us remind that a subset $A$ of a space $X$ is called regular closed if ii is the closure of some open set $U \subset X$. The Stone - Čech remainder $\beta \omega \backslash \omega$ we denote by $\omega^{\star}$.

3.9. Remark. Proposition 2.4 yields that open and regular closed subsets of a weakly separable space are weakly separable. But it cannot be extended to neither $G_{\delta}$-subsets nor finite intersections of regular closed subsets. As a counterexample one can take a separable compactum $X=X_{0} \cup X_{1}$ such that $X_{i}$ are regular closed in $X$, and $X_{0} \cap X_{1}$ is a non-separable $G_{\delta}$-set in $X$. Such a compactum $X$ can be obtained from $\beta \omega \times\{0,1\}$ by the identification of the sets $\omega^{\star} \times\{0\}$ and $\omega^{\star} \times\{1\}$.

For non-compact spaces, we can modify Examples 3.2 and 3.4 in the following way.

3.10. Example. There exists a separable, countably compact, Tychonoff space $X$ containing open, countably compact, disjoint subsets $U_{0}$ and $U_{1}$ such that the intersection $\bar{U}_{0} \cap \bar{U}_{1}$ is weakly separable, but non-separable.

Proof. Let $Y_{i}^{\prime}=\beta \omega \times\{i\}, i=0,1$. Fix an arbitrary point $a \in \omega^{\star}$. By $Y$ we denote a quotient space that we get from $Y^{\prime}=Y_{0}^{\prime} \cup Y_{1}^{\prime}$ by identification of the points $a \times\{0\}$ and $a \times\{1\}$. Let $f: Y^{\prime} \rightarrow Y$ be the quotient mapping, $b=f(a \times\{i\}), Y_{i}=f\left(Y_{i}^{\prime}\right)$. Finally, let $X$ be a subset of the product $Y \times D^{\omega_{1}}$ that is a disjoint sum of the sets $U_{0},\{b\} \times G, U_{1}$, where $U_{i}=\left(Y_{i} \backslash\{b\}\right) \times D^{\omega_{1}}$ and $G$ is a space from Example 3.2.

Clearly, $U_{i}$ are open in $X$ and $\bar{U}_{0} \cap \bar{U}_{1}=\{b\} \times G$. Further, $Y_{i} \backslash\{b\}$ is homeomorphic to the space $\beta \omega \backslash\{a\}$ that is countably compact according to 
([9], Example 3.10.18). Hence, $U_{i}$ is countably compact as a product of the countably compact space $Y_{i} \backslash\{b\}$ and the compact space $D^{\omega_{1}}$. Moreover, $U_{i}$ is separable as a product of separable spaces. Thus, $X$ is separable, since $U_{0} \cup U_{1}$ is dense in $X$. At last $X$ is countably compact as a union of countably compact spaces $U_{0}, U_{1}$ and $\{b\} \times G$.

3.11. Example. There exists a separable, $\sigma$-compact, normal space $X$ containing open, $\sigma$-compact, disjoint subsets $U_{0}$ and $U_{1}$ such that the intersection $\bar{U}_{0} \cap \bar{U}_{1}$ is weakly separable, but non-separable.

Proof. Let $X$ be a subset of the product $[-1,1] \times D^{\omega_{1}}$ that is a disjoint sum of the sets $U_{0},\{0\} \times H, U_{1}$, where $U_{0}=[-1,0) \times D^{\omega_{1}}, U_{1}=(0,1] \times D^{\omega_{1}}$ and $H$ is the space from Example 3.4. All declared properties of $X$ can be verified by scheme of the previous proof.

\section{On weakly separable spaces and some functorial construc- tions}

We are going to recall some notions and facts concerning hyperspaces and related spaces. For a topological space $X$, by $\exp X$ one denotes the set of all non-empty closed subsets of $X$. Let $U_{1}, \ldots, U_{k}$ be a finite family of open subsets of $X$. Set

$$
O<U_{1}, \ldots, U_{k}>=\left\{F \in \exp X: F \subset \cup_{1}^{k} U_{i}, F \cap U_{i} \neq \emptyset, i=1, \ldots, k\right\} .
$$

By the hyperspace of closed subsets of $X$ we call the set $\exp X$ that is equipped with so called (finite) Vietoris topology, whose open base consists of sets $O<$ $U_{1}, \ldots, U_{k}>$, where $U_{i}$ are open in $X$.

Now we indicate certain prominent subspaces of $\exp X$. The first of them is a hypersymmetric n-product $\exp _{n} X$ of a space $X$, where $n$ is a positive integer. It consists of no more than $n$-point closed subsets of $X$. The second one is:

$$
\exp _{\omega} X=\cup\left\{\exp _{n} X: n=1,2, \ldots\right\} .
$$

At last, $\exp _{c} X$ consists of all non-empty compact closed subsets of $X$. For an arbitrary space $X$ the following inclusions take place:

$$
\exp _{n} X \subset \exp _{\omega} X \subset \exp _{c} X \subset \exp X .
$$

If $X$ is a $T_{1}$-space, then it is naturally homeomorphic to $\exp _{1} X$. The following assertions are due to E.Michael [10].

4.1. Proposition. If $X$ is a $T_{1}$-space, then:

1) $\exp X$ is a $T_{1}$-space; 
2) $\exp _{\omega} X$ is dense in $\exp X$.

4.2. Proposition. If $X$ is a $T_{1}$-space, by assigning to $\left(x_{0}, \ldots, x_{n-1}\right) \in X^{n}$ the point $\left\{x_{0}, \ldots, x_{n-1}\right\} \in \exp _{n} X$ we obtain a continuous surjection $\pi_{n, X} \equiv$ $\pi_{n}: X^{n} \rightarrow \exp _{n} X$.

Now let $G$ be a subgroup of the symmetric group $S_{n}$ of all permutations of the set $n=\{0, \ldots, n-1\}$. For an arbitrary space $X$, the group $G$ acts on $X^{n}$ by:

$g\left(x_{0}, \ldots, n-1\right)=\left(x_{g(0)}, \ldots, x_{g(n-1)}\right.$ for every $g \in G$.

Let $S P_{G}^{n} X$ be the quotient space $X^{n} / G$, and let $\pi_{G, X}^{n} \equiv \pi_{G}^{n}: X^{n} \rightarrow$ $S P_{G}^{n} X$ be the quotient mapping. The space $S P_{G}^{n} X$ is called the $G$-symmetric $n$-product of $X$.

4.3. Proposition. If $X$ is a $T_{1}$-space, then $S P_{G}^{n} X$ is a $T_{1}$-space as well.

In fact, $X^{n}$ is a $T_{1}$-space, and $S P_{G}^{n} X$ is an image of $X^{n}$ under the quotient mapping $\pi_{G}^{n}$ whose fibers are finite.

Clearly, there is a unique mapping $\pi_{n}^{G}: S P_{G}^{n} X \rightarrow \exp _{n} X$ such that

$$
\pi_{n}=\pi_{n}^{G} \circ \pi_{G}^{n} .
$$

4.4. Proposition. If $X$ is a $T_{1}$-space, then the mapping

$$
\pi_{n}^{G}: S P_{G}^{n} X \rightarrow \exp _{n} X
$$

is continuous.

Proof. From Proposition 4.2 and equality (4.2) we get that the continuous mapping $\pi_{n}$ is a composition of the quotient mapping $\pi_{G}^{n}$ and the mapping $\pi_{n}^{G}$. In this situation $\pi_{n}^{G}$ is continuous.

4.5. Theorem. For an arbitrary $T_{1}$-space $X$, positive integer $n$, and a group $G \subset S_{n}$, the following conditions are equivalent:

1) $X$ is weakly separable;

2) $X^{n}$ is weakly separable;

3) $S P_{G}^{n} X$ is weakly separable;

4) $S P^{n} X$ is weakly separable;

5) $\exp _{n} X$ is weakly separable;

6) $\exp _{\omega} X$ is weakly separable;

7) $\exp _{c} X$ is weakly separable;

8) $\exp X$ is weakly separable.

Proof. Implications 1) $\rightarrow \cdots \rightarrow 8$ ) are just immediate corollaries of preliminary results: 
1) $\rightarrow 2$ ) (Proposition 2.4.4); 2) $\rightarrow 3$ ) (Proposition 2.4.6); 3) $\rightarrow 4$ ) (trivial); $4) \rightarrow 5$ ) (Propositions 2.4.6 and 4.4); 5) $\rightarrow 6$ ) (Proposition 2.4.5); 6) $\rightarrow 7$ ) $\rightarrow 8$ ) (Propositions 2.4.3 and 4.1, and inclusions (4.1)). Let us check that 8) implies 1). Consider the family

$$
O(\mathcal{T})=\{O<U>: U \in \mathcal{T}(X)\} .
$$

By Proposition 2.2.4 there exist families $T_{i} \subset \mathcal{T}(X), i \in \omega$, such that

$$
\mathcal{T}(X)=\cup\left\{T_{i}: i \in \omega\right\}
$$

and every family

$$
O\left(T_{i}\right)=\left\{O<U>: U \in T_{i}\right\}
$$

is centered. But, clearly, $O\left(T_{i}\right)$ is centered if and only if $T_{i}$ is centered. Applying Proposition 2.2.4 once more we conclude that $X$ is weakly separable. Theorem 4.5 is proved.

The assertion of Theorem 4.5 cannot be extended to the class of $T_{0^{-}}$ spaces.

4.6. Example. There exists a separable $T_{0}$-space $X$ such that $\exp _{1} X$ is not weakly separable.

Take an arbitrary uncountable set $X$ and fix some its point $x_{0}$. Topology on $X$ is defined as follows. A non-empty set $U \subset X$ is open iff $x_{0} \in U$. On one hand, $X$ contains a dense set consisting of one point $x_{0}$. On the other hand, $\exp _{1} X=X \backslash\left\{x_{0}\right\}$ is a discrete uncountable set.

4.7. Question. Is it true that $\exp X$ is (weakly) separable for every countable space $X$ ?

Let us recall that an uncountable cardinal number $\tau$ is said to be a (pre)calibre of a space $X$ if every family $u$ of cardinality $\tau$ consisting of nonempty open subsets of $X$ contains a subfamily $u_{0}$ with non-empty intersection ( $u_{0}$ is centered). The following assertions are evident and well known.

4.8. Proposition. If $X$ is separable, then $\omega_{1}$ is a calibre of $X$.

4.9. Proposition. If $\omega_{1}$ is a precalibre of $X$, then $X$ has the Souslin property, i.e. every family of pairwise disjoint non-empty open subsets of $X$ is countable.

4.10. Proposition. If $Y \subset X$ is dense in $X$ and $\tau$ is a precalibre of $X$, then $\tau$ is a precalibre of $Y$.

Shanin got much stronger result than Proposition 4.8. We give here an important corollary of it.

4.11. Theorem [13]. If $X_{\alpha}$ is separable for every $\alpha \in A$, then $\omega_{1}$ is a calibre of the product $\Pi\left\{X_{\alpha}: \alpha \in A\right\}$. 
This theorem can be generalized in the following way.

4.12. Theorem. If $X_{\alpha}$ is weakly separable for every $\alpha \in A$, then $\omega_{1}$ is a precalibre of the product $X=\Pi\left\{X_{\alpha}: \alpha \in A\right\}$.

Proof. In accordance with Theorem 2.5 there exists a separable extension $e X_{\alpha}$ for each $\alpha$. Let $e X=\Pi\left\{e X_{\alpha}: \alpha \in A\right\}$. Then $\omega_{1}$ is a calibre of $e X$ by Theorem 4.11. Hence, $\omega_{1}$ is a precalibre of $X$ in view of Proposition 4.10.

From Proposition 4.9 and Theorem 4.12 we get

4.13. Corollary [2] If $X_{\alpha}$ is weakly separable for every $\alpha \in A$, then the product $\Pi\left\{X_{\alpha}: \alpha \in A\right\}$ has the Souslin property.

4.14. Remark. The space $G$ from Example 3.2 shows that $\omega_{1}$ has not to be a calibre of a weakly separable space (even a normal countably compact group). Indeed, $G$ is a union of an increasing sequence $\left\{D^{\alpha}: \alpha<\omega_{1}\right\}$ of nowhere dense compacta. Then $\left\{U_{\alpha}=G \backslash U_{\alpha}: \alpha<\omega_{1}\right\}$ is a decreasing sequence of dense open sets with empty intersection.

4.15. Proposition. Let $X_{\alpha}$ consist of more than one point, $\alpha \in A$, and let $X=\Pi\left\{X_{\alpha}: \alpha \in A\right\}$. Then the following conditions are equivalent:

1) $X$ is weakly separable;

2) $\operatorname{card}(A) \leq 2^{\omega}$;

3) $X$ is separable.

Proof. Let $X$ be weakly separable. Then $b X=\Pi\left\{\beta X_{\alpha}: \alpha \in A\right\}$ is a separable compactum by Theorem 2.3 and Proposition 2.4.3. According to Pondiczery - Marczewski theorem (look at [9], Exercise 2.3.G), $\operatorname{card}(A) \leq 2^{\omega}$. Then $X$ is separable as a product of $2^{\omega}$ separable spaces ([9], Theorem 2.3.15). Proposition 4.15 is proved.

\section{On the weak separability of $C_{p}(X)$}

In this section all spaces are Tychonoff. For a space $X$, by $C_{p}(X)$ we denote the space of all continuous realvalued functions equipped with a pointwise convergence topology. In this topology $C_{p}(X)$ is a dense subset of the Tychonoff product $\mathbf{R}^{X}$.

Let us also recall that a cardinal number $\tau$ is said to be an $i$-weight of a space $X$ (written $\tau=i w(X)$ ) if $\tau$ is the smallest cardinal number such that there is a one-to-one continuous mapping $f: X \rightarrow Y$ onto a space $Y$ of weight $\tau$. In particular, if $X$ is a compactum, then $i w(X)=w(X)$.

5.1. Theorem [11]. For an arbitrary $X$ we have

$$
d\left(C_{p}(X)=i w(X) .\right.
$$


5.2. Corollary. For an arbitrary non-metrizable compactum $X$ the space $C_{p}(X)$ is non-separable.

Now we characterize spaces $X$ with weakly separable $C_{p}(X)$,

5.3. Theorem. For a space $X$ the following conditions are equivalent:

1) $C_{p}(X)$ is weakly separable;

2) $\mathbf{R}^{X}$ is weakly separable;

3) $\operatorname{card}(X) \leq 2^{\omega}$;

4) $\mathbf{R}^{X}$ is separable.

Proof. We follow the next scheme: 1$) \rightarrow 2$ ) $\rightarrow 3$ ) $\rightarrow$ 4) $\rightarrow$ 1) using Propositions $2.4(3), 4.15$, and 2.4(2) respectively.

5.4. Example. There exists a compactum $X$ such that $C_{p}(X)$ is weakly separable but non-separable.

For $X$ we can take an arbitrary non-metrizable compactum of cardinality $\leq 2^{\omega}$. In fact, in this situation Corollary 5.2 and Theorem 5.3 provide the required properties of $C_{p}(X)$.

\section{References}

[1] A.V. Arhangel'skii: On one class of spaces containing all metric and all locally compact spaces, Matem. Sbornik 67:1 (1965), 55-85.

[2] R.B. Beshimov: On some properties of weakly separable spaces, Uzbek. Math. Journ. 1 (1994), 7-11 (in Russian).

[3] R.B. Beshimov: A weakly separable space and separability, Doklady Uzbek. Akad. Nauk 3 (1994), 10-12 (in Russian).

[4] R.B. Beshimov: Covariant functors and weak separability, Doklady Uzbek. Akad. Nauk 7 (1994), 9-11 (in Russian).

[5] R.B. Beshimov: Weakly separable spaces and their separable compactifications, Doklady Uzbek. Akad. Nauk 1 (1997), 15-18 (in Russian).

[6] W.W. Comfort: An example on density character, Arch. Math. 14 (1963), 422423.

[7] H.H. Corson: Normality in subsets of product spaces, Amer. J. Math. 81 (1959), 785-796.

[8] E.K. van Douwen: Density of compactifications, In: Set-theoretic Topology, Academic Press, 1977, 97-110.

[9] R. Engelking: General Topology, Warszawa, 1977. 
[10] E. Michael: Topologies on spaces of subsets, Trans. Amer. Math. Soc. 71 (1951), $152-182$.

[11] N. Noble: The density character of function spaces, Proc. Amer. Math. Soc. 44:2 (1974), 228-233.

[12] L.S. Pontryagin: Continuous Groups, Moscow, 1938.

[13] N.A. Shanin: On the product of topological spaces. Trudy Steklov Inst. 24, Moscow, 1948.

Tashkent State Pedagogical Institute Nizami

Tashkent, Uzbekistan

Received February 10, 2002 